\title{
Optimisation multi-critère pour véhicules autonomes en environnement dynamique
}

\section{Multi-criteria optimization for autonomous vehicles in a dynamic environment}

\author{
Jean-Baptiste Receveur ${ }^{1}$, Pierre Melchior ${ }^{2}$, Stéphane Victor ${ }^{2}$ \\ 1-2IMS - UMR 5218 CNRS, Université de Bordeaux/2Bordeaux INP, \\ 351 Cours de la Libération, 33405 Talence CEDEX, France \\ (e-mail : prenom.nom@ims-bordeaux.fr).
}

RÉSUMÉ. Dans les dernières années, la recherche sur les véhicules autonomes a connu un essor sans précédent. De plus, elle vient s'ajouter à un effort permanent des constructeurs pour diminuer la consommation énergétique de leurs véhicules pour qu'ils soient toujours plus économes pour les conducteurs. Cet article traite le sujet de l'optimisation de trajectoire pour un véhicule autonome de type automobile, basée sur des critères de consommation, de temps de trajet ou de confort. L'article se concentre principalement sur les tests de ces critères, et sur la méthode utilisée pour effectuer l'optimisation en combinant algorithme génétique et champs de potentiels. En cherchant des points intermédiaires optimaux dans le champ de potentiel, et en considérant de façon intelligente le mouvement des obstacles et le volume du véhicule, une trajectoire optimale peut être générée. Dans les deux premières parties de cet article les méthodes utilisées et le problème à résoudre sont décrits, puis quelques tests sur des scénarios réalistes sont effectués.

ABSTRACT. In the last few years much effort has been made towards more autonomous vehicles and fuel consumption reduction. This article deals with the issue trajectory optimization of unmanned terrestrial vehicles so as to reduce consumption, travel time or to improve comfort. Main focuses are set on testing different criteria and the possibility of using a genetic algorithm to improve the potential field methods. The main idea of this article is that potential field methods could be improved by adding a dynamic target in it. Two improvements are brought to the potential field method : the generation of an optimal path in the environment, and the joint generation an optimal motion.

MOTS-CLÉS. Véhicules autonomes, Planification de trajectoire, Champs de potentiel, Trajectoire optimale, Optimisation, Algorithmes génétiques, Dérivée fractionnaire.

KEYWORDS. Autonomous vehicles, Path planning, Potential fields, Optimal trajectory, Optimization, Genetic algorithms, Fractional differentiation.

\section{Introduction}

En robotique, la notion de chemin est fréquemment utilisée pour décrire la trajectoire spatiale que suit un robot d'un point à un autre. Dans la plupart des méthodes, la planification du chemin est découplée de celle du mouvement le long de ce chemin. Dans cet article, l'objectif est de planifier une trajectoire - chemin et mouvement le long de ce chemin - de façon couplée, dans un environnement dynamique, et de sorte que le véhicule puisse toujours réagir en cas d'événement imprévu. Le véhicule sera appelé Ego-Véhicule (EGV) pour le distinguer des autres véhicules.

En planification de chemin pour les véhicules, de nombreuses études se sont concentrées sur le fait de trouver des chemins courts ou évitant les obstacles (voir [Pruski, 1996], [Latombe, 2012]). Cependant, le caractère énergétique, dépendant surtout du mouvement, est une préoccupation relativement récente (voir [Schouwenaars et al., 2001]), que l'on retrouve dans des études parlant d'éco-conduite (voir [Wang and Cassandras, 2013], [Mensing et al., 2014]) ou de profil de vitesse pour les trains (voir [Wang et al., 2011], [Feng et al., 2014]). Dans ces derniers, la notion de chemin n'est pas nécessaire puisque les rails fixent le chemin. Seul le mouvement est considéré. Le caractère autonome ainsi que les dépenses énergétiques 


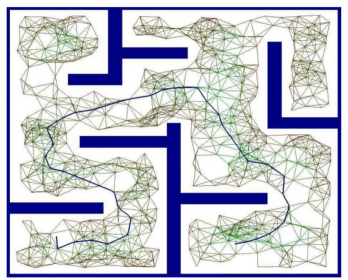

(a)

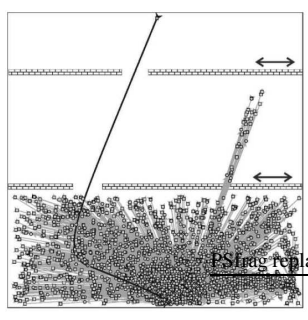

(b)

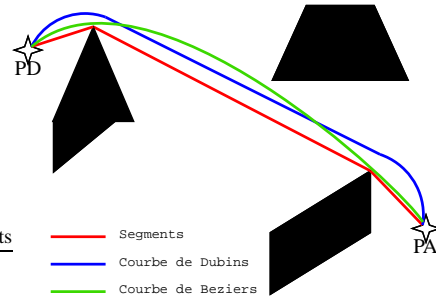

(c)

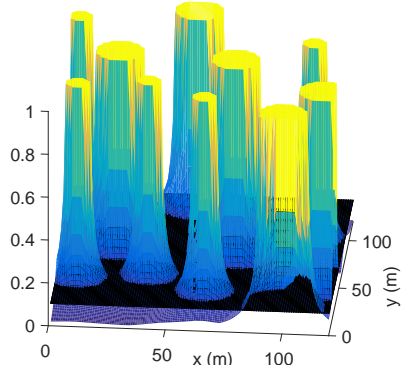

(d)

Figure 1. (a) Décomposition en cellules (b) Algorithme $A$ (c) Segments, courbes de Dubins et de Béziers (d) Champs de potentiel artificiels ou Artificial Potential Fields (APF)

sont un problème présent également dans le domaine aérien avec les drones (voir [Capello et al., 2008]). Les valeurs utilisées ici sont celles d'une automobile.

Les contributions principales de cette étude sont la recherche de différents critères, tels que l'énergie ou le confort, et leur inclusion dans la planification de trajectoire. Une méthode globale, l'algorithme génétique (GA), et une méthode locale, les champs de potentiel (APF), sont astucieusement combinés pour former une méthode de planification optimale et réactive. La nouveauté vient également d'une prise en compte astucieuse de l'encombrement du véhicule ainsi que du mouvement des obstacles. Cette prise en compte est très intéressante pour un scénario réaliste tel qu'un carrefour. De plus, la prise en compte du mouvement des obstacles sans recalculer complètement le champ de potentiel rend l'algorithme plus rapide, plus réactif.

En section 1., un bref état de l'art des méthodes de planification est donné. En section 2., le problème de planification est décrit, ainsi que les méthodes pour le résoudre en sections 3. et 4.. Enfin, quelques simulations sont montrées en section 5. ainsi qu'une conclusion en section 6 .

\section{1. État de l'art en planification de trajectoire}

Les méthodes de planification de trajectoire sont souvent divisées en deux catégories : d'un côté les méthodes globales (hors-ligne), méthodes historiquement basées sur la théorie des graphes, et de l'autre côté les méthodes locales (en-ligne), trouvant la meilleure position suivante pour l'EGV. Mais la classification est devenue plus compliquée et on pourrait aujourd'hui représenter l'ensemble des méthodes de classification sur plusieurs axes : l'axe local-global, l'axe chemin-trajectoire, l'axe discret-continu, ou encore l'axe déterministe-heuristique. Un résumé non-exhaustif est donné dans le Tableau 1.1 séparant seulement les méthodes globales-locales et les méthodes déterministes-heuristiques. Quelques illustrations de méthodes sont données en Figure 1.

Dans l'équipe CRONE, les champs de potentiel ont été étudiés (voir [Melchior et al., 2007]). Cette méthode locale, bien que rapide et élégante mathématiquement, présente quelques inconvénients importants : les minima locaux peuvent mener le véhicule vers la mauvaise destination ou le bloquer. De plus, comme pour la plupart des méthodes locales, une trajectoire obtenue par les APF (Artificial Potential Fields) est difficile à optimiser, puisqu'elle est définie par la forme du potentiel. Pour résoudre chacun de ces problèmes, une nouvelle manière de définir le potentiel attractif sera proposée en Section 3.1.. Par 


\begin{tabular}{|c|c|c|c|c|}
\hline \multicolumn{5}{|c|}{ Planification de trajectoire ou de chemin, discrète ou continue } \\
\hline \multicolumn{3}{|c|}{$\begin{array}{l}\text { Algorithmes hors-ligne (ou globaux), Avantages : } \\
\text { optimisable, Inconvénients : obstacles fixes (ou mouvement } \\
\text { présupposé), calcul plus long }\end{array}$} & \multicolumn{2}{|c|}{$\begin{array}{l}\text { Algorithmes en-ligne (ou } \\
\text { locaux), Avantages : } \\
\text { rapides, réactifs,... } \\
\text { Inconvénients : } \\
\text { difficilement optimisables }\end{array}$} \\
\hline $\begin{array}{l}\text { Méthodes } \\
\text { déterministes }\end{array}$ & Méthodes h & ristiques & $\begin{array}{l}\text { Méthodes } \\
\text { déterministes }\end{array}$ & $\begin{array}{l}\text { Méthodes } \\
\text { heuristiques }\end{array}$ \\
\hline $\begin{array}{l}\text { Utilisant } \\
\text { l'intégration des } \\
\text { équations : basées } \\
\text { sur les méthodes } \\
\text { Lagrangiennes, les } \\
\text { splines, les courbes } \\
\text { de Béziers ([Choi } \\
\text { and Elkaim, 2008]) }\end{array}$ & $\begin{array}{l}\text { Algorithmes } \\
\text { d'optimisation : } \\
\text { Algorithmes } \\
\text { génétiques (GA), } \\
\text { Essaims particulaires, } \\
\text { Colonies de fourmis } \\
\text { ([Collette and Siarry, } \\
\text { 2002], [Besada-Portas } \\
\text { et al., 2013]) }\end{array}$ & $\begin{array}{l}\text { Utilisant une } \\
\text { approche feuille } \\
\text { de route : graphe } \\
\text { de visibilité, } \\
\text { autoroute, } \\
\text { décomposition en } \\
\text { cellules, Voronoi } \\
\text { ([Latombe, } \\
\text { 2012]) + } \\
\text { Dijkstra, } A, A^{*} \\
\text { ([Nilsson, 1969]) }\end{array}$ & $\begin{array}{l}\text { Champs de } \\
\text { potentiel } \\
\text { artificiels } \\
\text { ([Khatib, } \\
\text { 1986]), His- } \\
\text { togrammes } \\
\text { de champs } \\
\text { de vecteurs, } \\
\text { Fenêtre } \\
\text { dynamique }\end{array}$ & $\begin{array}{l}\text { Tentacules } \\
\text { ([Alia et al., } \\
\text { 2015]), Cônes } \\
\text { de collision }\end{array}$ \\
\hline
\end{tabular}

Tableau 1.1. Tableau récapitulatif des différentes méthodes de planification

ailleurs, dans de nombreuses méthodes de planification, la notion de points intermédiaires est présente. Des fonctions splines sont utilisées ensuite pour faire le lien entre les points intermédiaires.

Dans cet article, deux méthodes sont utilisées pour optimiser et suivre la trajectoire, respectivement un algorithme génétique et les champs de potentiel. Par rapport au Tableau 1.1, la méthode proposée combine à la fois une méthode globale (GA), qui apporte de l'optimalité sur le moyen terme, et une méthode locale $(\mathrm{PF})$, qui permet de réagir rapidement aux événements imprévus. La combinaison proposée "points intermédiaires optimaux $(\mathrm{GA})$ + splines + réaction par le PF" est inédite.

\section{Définition du problème}

\subsection{Environnement de I'EGV}

\subsubsection{Obstacles :}

L'EGV peut rencontrer différents obstacles sur son chemin décrits en section 3.3.. Les bords de route, voitures, ou vélos sont représentés par des obstacles rectangulaires, alors que les piétons et autres obstacles seront représentés par des cercles. Sur les différentes figures, les obstacles eux-mêmes seront représentés en noir et les zones de danger les entourant en gris (voir [Poty, 2006] et les Figures 2 et 3 ). Ces zones sont représentées en rouge lorsque l'EGV les touche. 


\subsubsection{Distance caractéristique :}

Dans le cas automobile, le trajet d'un véhicule peut être divisé en trois catégories :

— le chemin à longue distance (de quelques centaines de mètres à kilomètres) est gérée par le GPS, qui planifie une route;

- le mouvement à courte distance (de un à dix mètres) est géré par les organes fonctionnels et réactifs du véhicule, par sa dynamique;

- la trajectoire pour des distances intermédiaires (d'une dizaine de mètre à un kilomètre) est une distance caractéristique pour un véhicule, gérée actuellement par le conducteur, et qui pourrait être gérée par un planificateur autonome.

Cent mètres est également un bon ordre de grandeur de la sensibilité maximum de certains capteurs (la sensibilité d'un capteur infrarouge par exemple). Entre les informations de cartes et les informations des capteurs, le véhicule autonome peut reconstruire de façon intéressante un environnement sur des distances intermédiaires. L'environnement est composé d'un point de départ noté PD (par exemple $(0,0))$ et d'arrivée notée PA (par exemple $(120,120)$ ). Dans la suite, les indices 0 et $f$ feront référence respectivement aux états initial et final.

\subsection{Variables d'optimisation}

Comme explicité en Section 1., de nombreuses méthodes utilisent la notion de points intermédiaires, et la méthode proposée sera basée sur l'optimisation de la position de tels points. Le nombre de points $K$ dépend fortement de l'environnement et son choix ne sera pas discuté ici. Ces points intermédiaires seront ensuite reliés entre eux par des splines, dans l'optimisation, de manière à évaluer des trajectoires composées de suffisamment de points dits d'interpolation pour être considérées comme continues. La distance considérée étant $120 \mathrm{~m}$, le théorème de Shannon sur l'échantillonnage affirmant que la période d'échantillonnage doit être deux fois plus faible que la plus petite période du signal, on prendra au moins $n_{\text {interp }}=240$ points pour l'interpolation, soit deux par mètre.

Les positions $\left(x_{0}, y_{0}\right)$ et $\left(x_{f}, y_{f}\right)$ sont connues, ainsi que $t_{0}=0$. Les variables d'optimisation seront ainsi les positions $\left(x_{k}, y_{k}\right)$ et temps $t_{k}$ intermédiaires, plus le temps final $t_{f}$ inconnu lui-aussi. Le nombre de variables d'optimisation est donc : $n_{\text {var }}=3 K+1$.

\subsection{Critères d'optimisation}

Un conducteur humain pourrait souhaiter une trajectoire non dangereuse, rapide, courte, écologique et confortable, certains de ces critères étant antagonistes. L'optimisation sera donc basée sur cinq critères à minimiser : danger, longueur et temps de trajectoire, énergétique, et inconfort. Dans les formules à suivre, $U_{r e p}$ est la matrice contenant les valeurs du champ de potentiel répulsif, $x x$ est l'abscisse de l'EGV, yy son ordonnée, $b_{1}$ et $b_{2}$ sont deux coefficients du véhicule (voir [Petit and Sciarretta, 2011]), $v$ et $a$ sont respectivement la vitesse et l'accélération de l'EGV, et jerk son jerk, la dérivée de l'accélération. $j$ est l'indice de la trajectoire interpolée, et $k$ celui des points intermédiaires optimaux. 
- Le critère de danger dépend uniquement des obstacles croisés dans l'environnement. Le danger peut être progressif, calculé par une fonction : $J_{\text {danger }}=\frac{1}{n_{\text {interp }}} \sum_{j} U_{r e p}\left(x x_{j}, y y_{j}\right)$, ou absolu : nul si aucun obstacle n'est rencontré, et très élevé si un obstacle est rencontré. Dans le premier cas, le danger peut être pondéré, dans le second c'est simplement un critère d'élimination de trajectoire. Dans les tests suivants, c'est la première méthode qui est retenue, et le champ de potentiel est utilisé comme marqueur du danger.

- Le critère de longueur de trajectoire s'exprime $J_{\text {longueur }}=\sum_{j} \sqrt{\left(x x_{j+1}-x x_{j}\right)^{2}+\left(y y_{j+1}-y y_{j}\right)^{2}}$, comme la somme des longueurs des segments entre les points interpolés.

- Le critère de temps $J_{\text {temps }}=t_{f}-t_{0}$ s'exprime comme la différence entre le temps final et le temps initial.

- Le critère de coût énergétique est issu de [Petit and Sciarretta, 2011] et s'exprime $J_{\text {energie }}=$ $\int_{0}^{t_{f}} b_{1} u(t) v(t)+b_{2} u(t)^{2} \mathrm{~d} t$. Cette expression est issue de l'expression de la puissance consommée

$$
P_{m}=b_{1} u v+b_{2} u^{2}, \text { où } b_{1}=\frac{\tau_{\max } R_{t}}{r}, b_{2}=\frac{R_{m}}{\kappa^{2}} \tau_{\max }^{2}
$$

avec $u=\frac{\tau}{\tau_{\max }}$ la variable de commande, $\tau$ le couple moteur, de valeur maximale $\tau_{\max }, R_{t}$ le rapport de transmission, $r$ le rayon de la roue, $R_{m}$ la résistance de l'armature moteur et $\kappa$ la constante de couple du moteur électrique. Cette expression est contrainte elle-même par le principe fondamental de la dynamique,

$$
\dot{v}=h_{1} u-h_{2} v^{2}-h_{0}-\gamma(x), \dot{x}=v
$$

où $h_{0}=g c_{r}, h_{1}=\frac{\tau_{\max } R_{t}}{M r}, h_{2}=\frac{c_{d} A_{f} \rho_{a}}{2 M}$ et $\gamma(x)=g \sin (\alpha(x))$ avec $c_{r}$ le coefficient de résistance au roulement, $g$ l'accélération de la pesanteur, $c_{d}$ le coefficient de résistance aérodynamique, $A_{f}$ l'aire apparente de face du véhicule, $\rho_{a}$ la densité de l'air, et $\alpha(x)$ l'angle de la route avec l'horizontale. L'énergie consommée est très liée à la pollution générée. Ce critère est basé sur la somme de deux puissances, intégrées sur le temps de parcours, donc deux énergies. La première puissance $b_{1} u(t) v(t)$ est une puissance utile, caractérisée par une force multipliée par une vitesse. La seconde puissance $b_{2} u(t)^{2}$ est une puissance perdue dans le moteur électrique, principalement par effet Joule. La somme de ces deux puissances représente la puissance totale consommée par le moteur qui, une fois intégrée, caractérise la consommation sur la trajectoire.

- Le critère de confort s'exprime $J_{\text {confort }}=J_{\text {conflong }}+J_{\text {conflat }}=\frac{1}{n_{\text {interp }}} \sum_{j}\left(j e r k_{j}^{u}+j e r k_{j}^{v}\right)$. Le jerk, dérivée de l'accélération, est souvent utilisé pour caractériser le caractère lisse d'une trajectoire. Le jerk ressentie par le passager est souvent responsable de son inconfort, qu'il soit latéral jerk $k^{v}$, longitudinal $j e r k^{u}$, ou vertical.

Il est à noter que la dynamique verticale du véhicule, comprenant en particulier l'adhérence, n'est pas prise en compte dans cette étude. La notion d'adhérence sera prise en compte dans une étude ultérieure sur un modèle complexe de véhicule.

Il est impossible d'atteindre un minimum absolu de tous ces critères avec une même trajectoire, ce qui est caractéristique de l'optimisation multicritère. Dans le cadre d'un véhicule autonome, le véhicule devra choisir une trajectoire, et non plusieurs. Il y a donc un choix des règles de priorité à définir. Les dilemmes que posent les critères choisis sont illustrés en Figure 2. Le problème est complexe : une part 


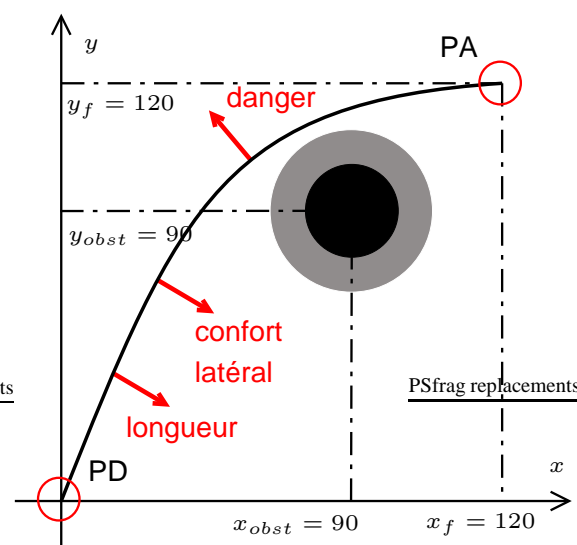

(a)

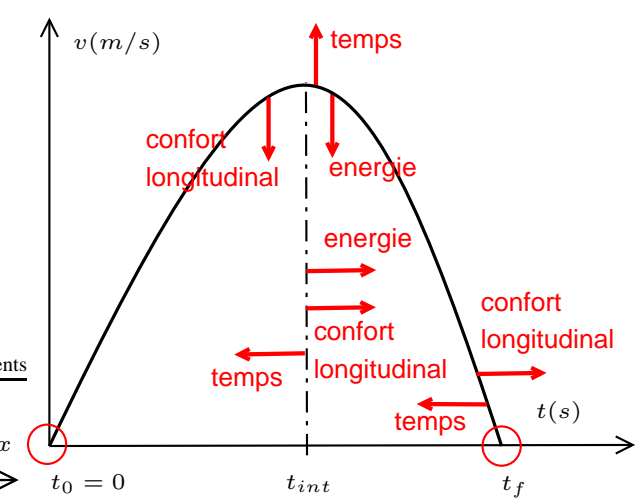

(b)

Figure 2. Dilemmes entre les critères

importante accordée à l'énergie ou au confort risquerait d'augmenter fortement le temps de trajet par exemple.

L'optimisation multicritère est un vaste domaine de recherche, et la méthode la plus simple de combinaison des critères est ici utilisée : l'agrégation pondérée. Un coefficient de pondération est attribué à chaque critère (voir Tableau 7.4), et le critère global sera la somme de ces critères pondérés. Le critère de danger est absolument prépondérant car la trajectoire ne peut pas être dangereuse. Plusieurs alternatives ont été proposées pour ces coefficients : différents modes de conduite peuvent être choisis par le conducteur, qui correspondent à différents jeux de pondération, accentuant l'un ou l'autre des coefficients. En cas de non choix, la longueur et le temps sont mis en avant, étant les critères GPS habituels, puis l'énergie et le confort. Cette priorisation est discutable, car le confort pourrait prendre une place plus importante dans l'optimisation. Enfin, l'optimisation par le GA peut générer une trajectoire idéale sans considération pour les cas d'urgence, car elle est couplée à une méthode locale réactive qui permet de gérer ces situations.

A partir de ces critères, dans l'environnement présenté, l'objectif est défini : trouver une trajectoire de $(0,0)$ à $(120,120)$ minimisant :

$$
J_{\text {global }}=\alpha_{\text {danger }} J_{\text {danger }}+\alpha_{\text {longueur }} J_{\text {longueur }}+\alpha_{\text {temps }} J_{\text {temps }}+\alpha_{\text {energie }} J_{\text {energie }}+\alpha_{\text {confort }} J_{\text {confort }} .
$$

\subsection{Encombrement de l'EGV}

Le critère de danger peut être calculé en utilisant seulement les points de la trajectoire, sans considérer le volume de l'EGV ou son orientation. Cependant, cela peut mener à de mauvais résultats comme montré en Figure 3 (a). Le volume peut être pris en considération dans le danger en ajoutant six points en plus du centre de gravité de l'EGV, comme illustré en Figure 3 (b). Les croix rouges sont les points pris en compte, de façon à éviter le contact entre l'EGV et les obstacles.

L’angle de lacet approché du véhicule $\psi=\operatorname{atan}\left(\frac{y_{j+1}-y_{j}}{x_{j+1}-x_{j}}\right)$ est illustré sur cette figure également. 


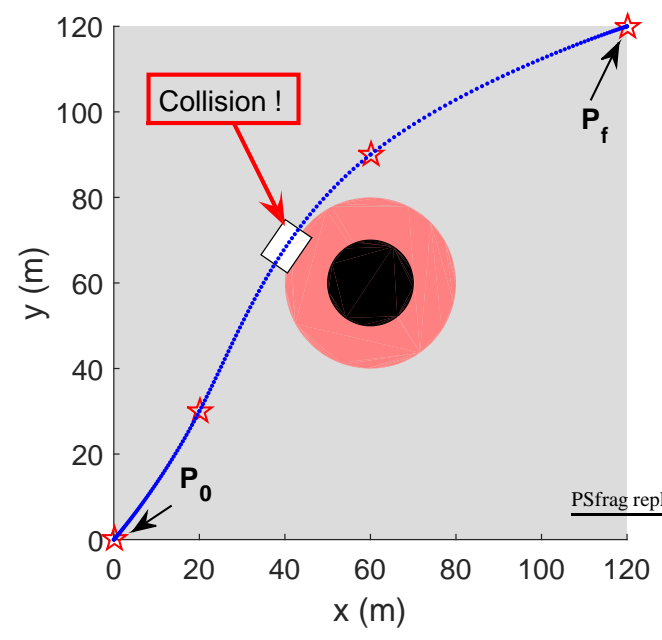

(a)

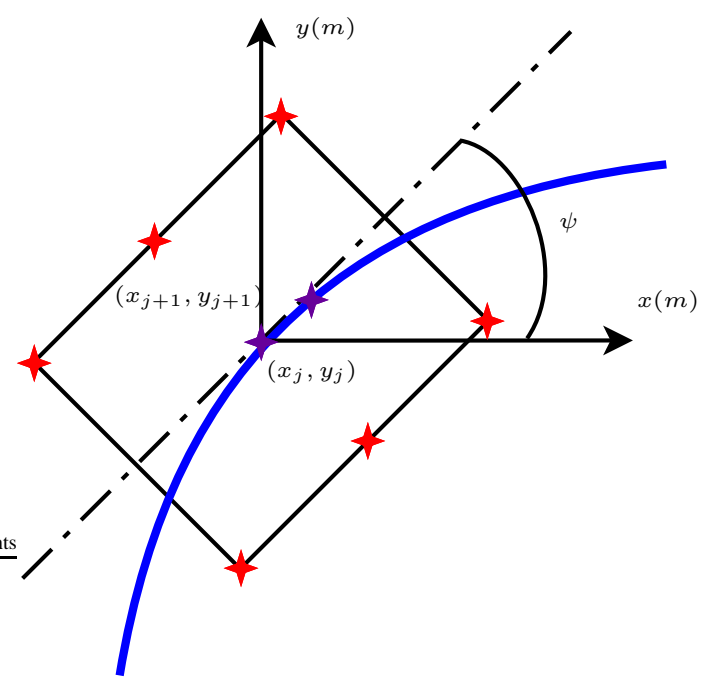

(b)

Figure 3. (a) Illustration des erreurs pouvant survenir en l'absence de prise en compte du volume (b) Volume de l'EGV et définition de $\psi$

\subsection{Contraintes d'optimisation}

Dans ce papier le véhicule est considéré comme une masse holonome aux dimensions horizontales du véhicule. Dans un second temps, il faudra considérer le véhicule avec toutes ses contraintes, notamment non-holonomes, ainsi que l'adhérence pneumatique/sol. L'algorithme est obligé de respecter des contraintes linéaires, qui sont :

$$
\begin{aligned}
& t_{0}<\ldots \quad t_{k}<\ldots \quad \ldots t_{f} \\
& 0<x, y<120 .
\end{aligned}
$$

Ces contraintes sont dites explicites et sont incontournables pour l'algorithme. D'autres contraintes dites implicites sont ajoutées. Elles sont des pénalités ajoutées aux critères, pour orienter la recherche. Par exemple, le critère de danger a été modifié de sorte que :

$$
J_{\text {danger }}=\frac{1}{n_{\text {interp }}} \sum_{j} U_{r e p}\left(x x_{j}, y y_{j}\right)+\operatorname{pen}\left(v_{j}\right)+\operatorname{pen}\left(a_{j}\right)
$$

où pen $\left(v_{j}\right)$ et pen $\left(a_{j}\right)$ sont des fonctions de pénalité dépendant de $v_{\max }$, la vitesse maximale autorisée pour le scénario (notion réglementaire), et $a_{\max }$ l'accélération maximale que peut fournir le moteur (notion technique). Ces contraintes tendent à faire respecter les limites plus ou moins strictement suivant la sévérité des fonctions de pénalité. Elles sont moins dommageables que les contraintes explicites non linéaires en termes de temps de calcul. Étant non contraignantes, elles conduisent cependant parfois à des solutions non acceptables, qu'il convient d'éliminer ou de signaler a posteriori. Les trajectoires étant dans un environnement routier, ces trajectoires non faisables sont donc rares (voir études statistiques ultérieures). Une fonction de pénalité pourrait être :

$$
\operatorname{pen}\left(v_{j}\right)=\left(\max \left(0, v_{j}-v_{\max }\right)\right)^{2},
$$

accentuant ainsi la pénalité de façon polynomiale en fonction de l'écart positif à la vitesse limite.

Une fois le problème de planification de trajectoire défini, la méthode de planification utilisant les champs de potentiel et les algorithmes génétiques est présentée. 


\section{Champs de potentiel artificiels (APF)}

La méthode des champs de potentiel artificiel consiste à remplir virtuellement l'environnement du robot avec un potentiel dans lequel l'EGV est attiré vers une cible et repoussé par les obstacles (voir, par ex. [Latombe, 2012]). Cette méthode déterministe est particulièrement appréciée pour son élégance mathématique.

Avec la méthode utilisée (voir [Ge and Cui, 2002]), deux types de potentiel seront développés : les potentiels répulsifs, à pente positive, représentant le danger autour des obstacles, et les potentiels attractifs, à pente négative, représentant l'objectif à atteindre par l'EGV.

\subsection{Champ de potentiel attractif}

Le potentiel attractif $U_{a t t}$ (voir [Ge and Cui, 2002])

$$
U_{a t t}(\vec{p}, \vec{v})=\alpha_{p}\left\|\vec{p}_{c i b l e}-\vec{p}\right\|+\alpha_{v}\left\|\vec{v}_{c i b l e}-\vec{v}\right\|,
$$

est défini comme une fonction de la position et de la vitesse relative de l'EGV par rapport à la cible, où $\vec{p}$ et $\vec{v}$ sont, respectivement, la position et la vitesse de l'EGV, et $\vec{p}_{c i b l e}$ et $\vec{v}_{c i b l e}$ celles de la cible. $\alpha_{p}$ et $\alpha_{v}$ sont des coefficients de pondération. Plus de détails sont donnés dans [Receveur et al., 2017]. La force est ensuite définie comme l'opposé du gradient du potentiel, et dépend donc également de la vitesse et de la position relative.

\subsection{Champ de potentiel répulsif}

Le champ de potentiel répulsif

$$
\begin{aligned}
& U_{n_{r e p}}(r)=G_{r e p} \frac{r^{n_{r e p}-2}-r_{\text {max }}^{n_{r e p}-2}}{r_{\text {min }}^{n_{r e p}-2}-r_{\max }^{n_{r e p}-2}} \quad \text { si } \quad n_{\text {rep }} \in\left[0,2[\cup] 2,+\infty\left[\text { et } r \in\left[r_{\text {min }}, r_{\text {max }}\right]\right.\right. \\
& U_{2}(r)=\frac{\ln \left(r_{\max }\right)-\ln (r)}{\ln \left(r_{\max }\right)-\ln \left(r_{\min }\right)} \quad \text { si } n_{\text {rep }}=2 \quad \text { et } r \in\left[r_{\text {min }}, r_{\text {max }}\right] \\
& U_{n_{\text {rep }}}(r)=0 \quad \text { si } r \notin\left[r_{\text {min }}, r_{\text {max }}\right]
\end{aligned}
$$

dépend de $r_{\min }$ qui est souvent le rayon de l'obstacle, et $r_{\max }$ le rayon de l'obstacle plus le rayon au-delà duquel on considère que l'obstacle n'a plus d'importance, et de $n_{r e p}$ l'ordre fractionnaire de l'obstacle, qui détermine une forme pour le potentiel. Cette définition se base sur la définition de Weyl de la dérivée fractionnaire (voir [Melchior et al., 2001]). Ensuite, un seuil est fixé entre 0 et 1, définissant le niveau acceptable de danger. La Figure 4 (a) illustre les formes de potentiel en fonction de l'ordre de l'obstacle. La Figure 4 (b) représente un exemple de potentiel répulsif pour un obstacle rond d'ordre $n_{\text {rep }}=1$, avec un seuil de 0.2 . A partir du potentiel, la force répulsive peut être calculée comme pour le potentiel attractif.

La méthode APF ne nécessite pas que les mouvements des obstacles soient connus a priori, et ne nécessite que la mesure en-ligne des informations des obstacles. Elle est, de plus, réactive quand un événement inattendu arrive, comme un obstacle croisant la trajectoire. Malgré ces avantages, elle présente 


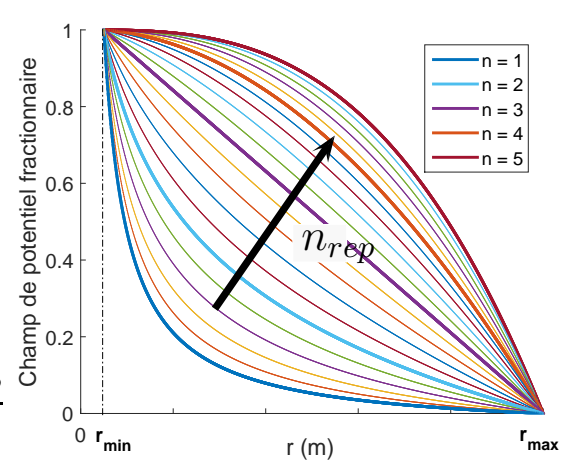

(a)

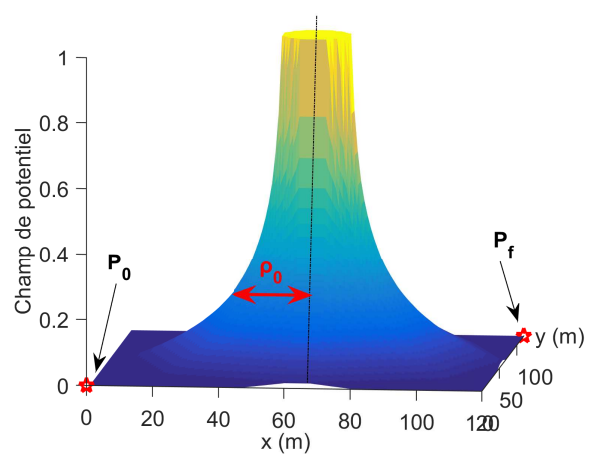

(b)

Figure 4. (a) Potentiel répulsif fractionnaire, l'abscisse $r=0$ étant le centre de l'obstacle, et $n=1$ correspondant au champ de potentiel coulombien (b) Potentiel répulsif pour un obstacle rond, avec un seuil de 0.2

l'inconvénient de ne pas pouvoir être optimisée facilement : les forces sont calculées à partir du gradient du potentiel, puis de ces forces est calculée l'accélération par la deuxième loi de Newton, puis par intégration, la vitesse est obtenue, puis la position de l'EGV, sans moyen de contrôle sur cette position, hormis la forme du potentiel.

\subsection{Choix de l'ordre des obstacles}

Comme explicité en Section 3.2., chaque obstacle a un ordre fractionnaire différent. Sur la Figure 4 la forme de la pente des différents obstacles est présentée, dépendant de $n_{\text {rep }}$. En sachant que la force exercée par l'obstacle est directement liée à la forme du potentiel, et que cette force devrait toujours augmenter lorsqu' on se rapproche de l'obstacle, l'ordre des obstacles ne devrait jamais excéder 3. L'ordre est donc considéré tel que $: n_{r e p} \in[0.1 ; 3]$.

Si l'ordre $n_{r e p_{k}}$ d'un obstacle $k$ représente sa dangerosité, il doit dépendre de trois éléments :

$$
n_{r e p_{k}}=f\left(\operatorname{Vuln}_{k}, \Delta v_{k}, v_{k}\right)
$$

où $V u l n_{k}$ est appelée la vulnérabilité, $\Delta v_{k}$ le potentiel de changement de vitesse, et $v_{k}$ la vitesse actuelle de l'obstacle. La fonction $f$ augmente avec chacun de ces paramètres, car ils augmentent le danger, et doit donner un résultat entre 0.1 (minimum arbitraire strictement positif) et 3 . La vulnérabilité peut être définie telle que :

$$
V u l n_{k}=V u l n_{k}^{e g o}+V u l n_{k}^{a l t e r}
$$

où $V u l n_{k}$ est arbitrairement entre 0 et 5 dépendant de $V u l n_{k}^{\text {ego }}, V u l n_{k}^{\text {alter }} \in[0 ; 2.5]$. Ces derniers sont, respectivement, la vulnérabilité de l'EGV considérant l'obstacle, et la vulnérabilité de l'obstacle considérant l'EGV. Les deux sont considérées comme également importantes dans cette étude, d'où leur influence identique sur la vulnérabilité totale $V u l n_{k}$.

Pour un véhicule automobile, différents types d'obstacles peuvent être envisagés, rassemblés dans le Tableau 3.2. De cette table, où $v_{\min }$ et $v_{\max }$ sont respectivement la vitesse minimum et la vitesse maximum que peut atteindre l'obstacle, le potentiel de variation de vitesse $\Delta v_{k}$ est défini tel que :

$$
\Delta v_{k}=\max \left(\left|v_{k}-v_{\min }\right|,\left|v_{k}-v_{\max }\right|\right) \text {. }
$$




\begin{tabular}{|l||l|l|}
\hline Type d'obstacle & {$\left[\mathbf{v}_{\mathbf{k}}^{\min }, \mathbf{v}_{\mathbf{k}}^{\max }\right](\mathrm{km} / \mathrm{h})$} & $\mathbf{V u l n}_{\mathbf{k}}$ \\
\hline Autre véhicule motorisé & {$\left[0 ; v_{l i m}\right]$} & $4=2+2$ \\
\hline Vélo, rollers, $\ldots$ & {$[0 ; 30]$} & $3=0.5+2.5$ \\
\hline Piéton & {$[0 ; 10]$} & $2.5=0+2.5$ \\
\hline Bords de routes, trottoirs & 0 & $0.5=0.5+0$ \\
\hline Signalisation, poteaux, murs & 0 & $2=2+0$ \\
\hline
\end{tabular}

Tableau 3.2. Vulnérabilité des obstacles $\left(V u l n_{k}=V u l n_{k}^{e g o}+V u l n_{k}^{\text {alter }}\right)$

La fonction $f$ est alors définie telle que :

$$
f\left(\operatorname{Vuln}_{k}, \Delta v_{k}, v_{k}\right)=\operatorname{Vuln}_{k}\left(A\left(v_{k}+\Delta v_{k}\right)+B\right)
$$

avec $f(0.5,0,0)=0.1 \Rightarrow B=0.2$ et $f\left(5, v_{\text {lim }}, v_{\text {lim }}\right)=3 \Rightarrow A=\frac{2}{5 v_{\text {lim }}}$, ce qui signifie que :

$$
f\left(\operatorname{Vuln}_{k}, \Delta v_{k}, v_{k}\right)=\operatorname{Vuln}_{k}\left(\frac{2}{5 v_{l i m}}\left(v_{k}+\Delta v_{k}\right)+0.2\right) .
$$

Cette formule permet au véhicule d'attribuer un ordre à n'importe quel type d'obstacle répertorié apparaissant dans l'environnement, dépendant de leur type, et de leur vitesse. D'autres améliorations pourraient être faites en prenant en compte la direction de l'obstacle.

Dans la partie suivante, la méthode des algorithmes génétiques et la façon dont elle viendra compléter la méthode des champs de potentiel seront expliquées.

\section{Algorithme génétique}

Les algorithmes génétiques, ou Genetic Algorithms en anglais (GA), permettent de résoudre des problèmes d'optimisation sans contraintes ou sous contraintes, en utilisant le principe de la sélection naturelle. Ils modifient de façon itérative une population d'individus qui sont des solutions potentielles au problème. A chaque itération, le GA choisit les meilleurs individus de la population pour en faire des $p a-$ rents pour la génération suivante. Les méthodes déterministes classiques comme la descente de gradient de critère ont tendance à buter sur des minima locaux, ou à ne pas converger tout court. Par exemple, si la fonction coût n'est pas continue ou pas différentiable, si certaines variables d'optimisation sont des entiers, les méthodes déterministes peuvent être complètement inefficaces.

Les algorithmes génétiques sont décrits plus en détails dans l'article [Receveur et al., 2017].

Comme dit en section 3., l'EGV est attiré vers la cible suivant la seconde loi de Newton, sous l'influence du potentiel attractif. L'EGV ne peut bouger que selon le champ de potentiel, ce qui signifie que, pour générer une trajectoire quasi-optimale, i.e. minimiser le critère d'optimisation [3], il faut modifier la forme du potentiel en conséquence. Une façon astucieuse de modifier le champ de potentiel est de placer une cible attractive dynamique de façon à ce que l'EGV soit toujours attiré au bon endroit au bon moment. Le rôle du GA est d'optimiser la trajectoire de cette cible.

Pour le GA, une trajectoire est représentée par un ensemble de points intermédiaires et de temps intermédiaires qui forment un individu. Le GA génère une fonction spline entre ces points, calcule le coût de la trajectoire ainsi formée, et après plusieurs générations choisi le meilleur individu, i.e. le meilleur 
jeu de points et temps intermédiaires, i.e. la trajectoire optimale. Le Tableau 4.3 représente un individu et correspond aux variables décrites en section 2.5.. $\left(x_{k}, y_{k}, t_{k}\right)$ sont respectivement l'abscisse, l'ordonnée et le temps de passage au $k$-ème point intermédiaire et $t_{f}$ est le temps nécessaire pour parcourir l'ensemble de la trajectoire. Pour illustrer le rôle du GA, des trajectoires exemples sont données en section 5 .

$$
\begin{array}{|l|l|l|l|l|l|l|l|}
\hline x_{1} & y_{1} & t_{1} & \ldots & x_{K} & y_{K} & t_{K} & t_{f} \\
\hline
\end{array}
$$

Tableau 4.3. Structure du chromosome pour la recherche de points optimaux

Le GA est amélioré par une initialisation au moyen de diagrammes de Voronoï. Quand les obstacles sont choisis comme centres du diagramme, les points d'intersection du diagramme sont souvent dans des zones libres de danger, bien que parfois proches des obstacles, et peuvent être utilisés comme population initiale de l'algorithme génétique. Cette population initiale, utilisée comme connaissance a priori, est trouvée très rapidement, et permet d'améliorer la vitesse de convergence de l'algorithme.

Dans la section suivante un ensemble de simulations est présenté, pour tester la méthode combinée ainsi que la prise en compte du volume du véhicule.

\section{Simulations et résultats}

Comme dit en section 4., l'algorithme génétique ne vient pas remplacer les champs de potentiels et leurs avantages. Ce n'est pas une méthode de planification en ligne, applicable en temps réel. Elle vient en complément de la méthode des potentiels en y ajoutant une cible dynamique optimale.

\subsection{Méthodes comparées}

L'objectif des scénarios de test est de comparer les résultats de deux méthodes :

— méthode 1 : méthode PF, champs de potentiel utilisés seuls, avec une cible placée directement à l'arrivée comme seul point d'attraction;

— méthode 2 : méthode GA-PF, champs de potentiel utilisés avec une cible dynamique optimale dont la trajectoire est générée par l'algorithme génétique.

Chacune des trajectoires issues de ces méthodes sera comparée avec la trajectoire optimale issue de l'algorithme génétique.

Pour inclure la mobilité des obstacles, le mouvement des obstacles est considéré comme permanent au moment de l'optimisation : la vitesse et l'accélération de ceux-ci sont considérées comme restant constantes. Deux scénarios sont utilisés, un cas simple avec un obstacle rond mobile (voir Figure 5), pour tester les réponses de base de l'algorithme (test avec un point intermédiaire en faisant varier la vitesse de l'obstacle pour vérifier quand on passe devant et derrière, comparaison avec une trajectoire de champs de potentiel simple), et un cas de franchissement de croisement un peu plus complexe (voir Figure 6), pour observer le comportement de l'EGV dans une situation réaliste. 


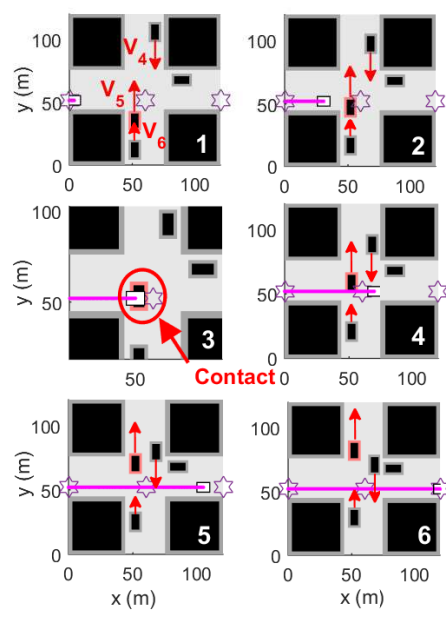

(a)

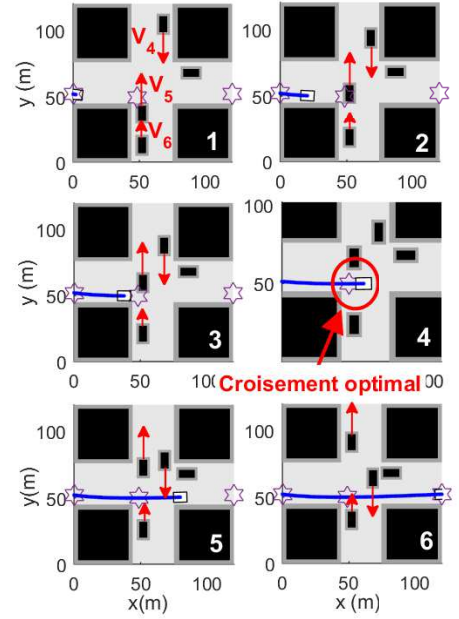

(b)
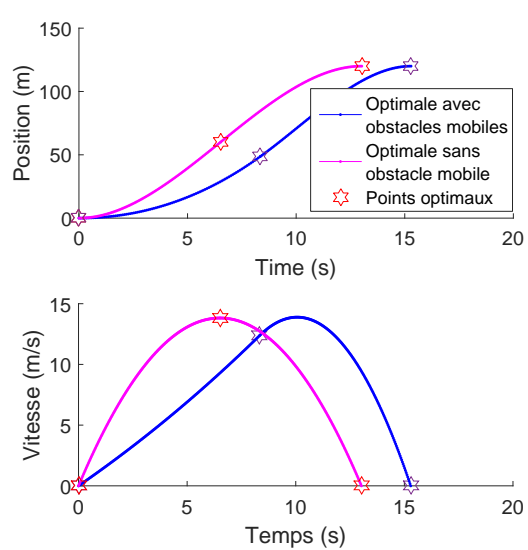

(c)

Figure 5. Scénario de croisement, comparaison entre (a) une trajectoire optimisée sans prise en compte du mouvement des obstacles et (b) une trajectoire optimisée avec prise en compte du mouvement des obstacles (c) consignes en position et vitesse correspondantes

Dans un premier temps, des trajectoires optimales seront présentées, sans et avec prise en compte du mouvement des obstacles. Dans un second temps, des trajectoires dans le champ de potentiel seront présentées, sans et avec optimisation, et seront comparées entre elles et avec la trajectoire optimale théorique. Dans les figures, les courbes d'abscisse en fonction du temps sont appelées "position", de façon à ne pas surcharger les figures en ajoutant les ordonnées.

\subsection{Trajectoires optimales en présence d'obstacles mobiles}

Dans un premier temps, seule la trajectoire optimale issue de l'algorithme génétique est considérée, sans notion de champ de potentiel, si ce n'est pour le calcul du danger. L'obstacle mobile est visible et identifié dès le début de la simulation. Sur la Figure 3, le volume de l'EGV n'avait pas été pris en compte. Sur la Figure 5 (a), la trajectoire est optimisée avec le GA pour un scénario de croisement réaliste, avec d'autres véhicules. Cependant, l'optimisation est faite sans considération du mouvement des obstacles, ce qui résulte en une collision possible de l'EGV avec un véhicule, ou en une trajectoire non optimale. Sur la Figure 5 (b), non seulement le mouvement des obstacles est pris en compte, mais également le volume du véhicule. Sur cette figure, il est visible que l'obstacle de vitesse $V_{5}$ est considéré par le GA comme allant vite, et donc il est préférable de passer derrière. L'EGV accélère plus lentement pour laisser le premier véhicule franchir le carrefour. L'EGV passe juste après ce premier véhicule, il attend le temps optimal nécessaire à ce que l'autre véhicule passe, sans avoir besoin de s'arrêter. Cela montre qu'il est important de prendre en compte le mouvement des obstacles, car cela peut donner des trajectoires bien plus intéressantes.

\subsection{Conséquences pour des trajectoires en-ligne dans les champs de potentiel}

Dans les études précédentes, les champs de potentiel étaient majoritairement utilisés pour permettre au véhicule d'éviter les obstacles en réagissant à leur présence. En considérant que les obstacles peuvent 


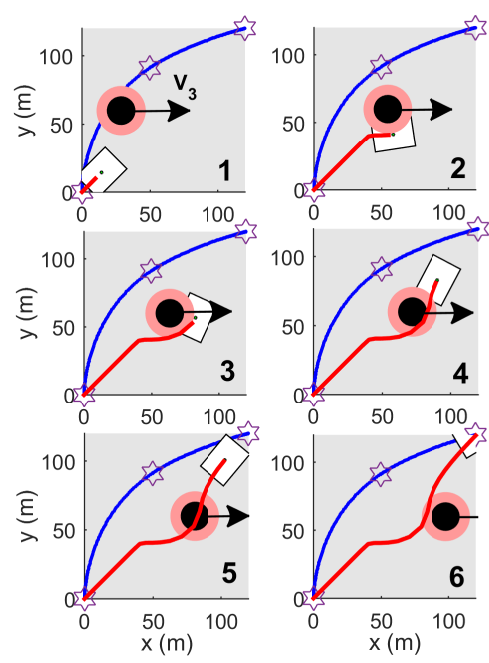

(a)
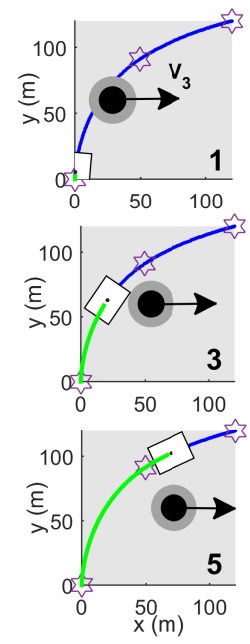

(b)
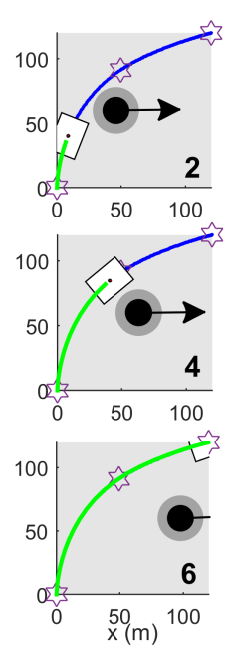
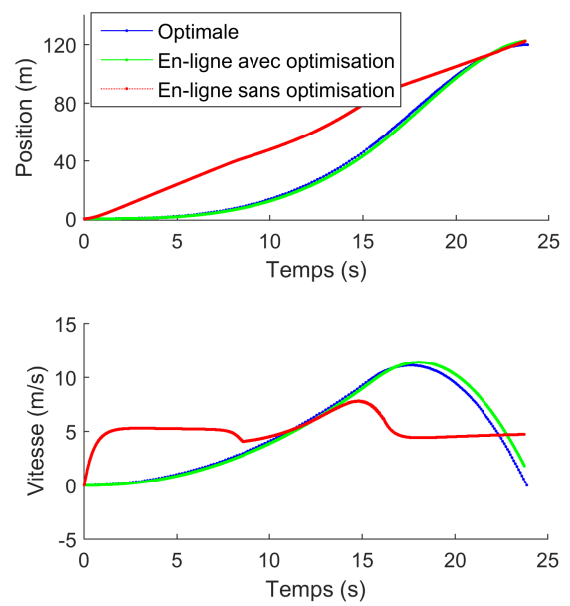

(c)

Figure 6. (a) Trajectoire en temps réel sans optimisation et (b) avec optimisation comparées à la trajectoire optimale (en bleu sur toutes les figures) pour un scénario simple (c) courbes de position et de vitesse en fonction du temps

garder le même mouvement qu'ils ont au début du calcul, la planification peut être améliorée par l'anticipation, en suivant une cible optimale dynamique, qui agit comme un minimum du potentiel se déplaçant de façon optimale.

La trajectoire optimale est calculée, puis une cible virtuelle est créée qui attire en permanence l'EGV vers un point donné, qui est un potentiel attractif comme donné en section 3.1.. La simulation illustrée en Figure 6 montre notamment l'impact de l'optimisation de la trajectoire par rapport à la trajectoire dans un champ de potentiel sans optimisation : la trajectoire optimale, la cible dynamique optimale, permet un passage optimisé derrière l'obstacle qui se déplace (b) alors que sans l'optimisation, l'EGV atteint l'obstacle et a du mal à l'éviter puisque celui-ci se déplace (a). Le critère de danger est proche de $10^{5}$ dans ce dernier cas, alors qu'il est nul dans le cas où l'EGV suit la cible dynamique optimisée.

Sur la Figure 7, la trajectoire optimale et la trajectoire en-ligne avec cible dynamique optimale sont données en (a), ainsi que les courbes correspondantes en position et en vitesse en (b). Sous ces figures sont données les valeurs du critère de temps calculé pour la trajectoire optimale $J_{\text {time }}^{g a}$ et pour la trajectoire enligne correspondante $J_{\text {time }}^{c p}$. La différence entre ces deux valeurs est de l'ordre de $0.3 \%$, ce qui correspond aux objectifs (moins de $1 \%$ de différence entre la trajectoire optimale et la trajectoire en-ligne). On note que cette trajectoire est $1.5 \mathrm{~s}$ plus longue que dans le cas où les obstacles ne sont pas censés bouger, ce qui correspond au temps nécessaire pour laisser passer le premier véhicule.

Les simulations réalisées confirment que la trajectoire issue de la méthode combinée GA-PF est très proche de la trajectoire optimale idéale. Cependant, contrairement à cette dernière, elle est modifiable en temps réel grâce à la présence du champ de potentiel, si un obstacle se déplace de façon inattendue. 

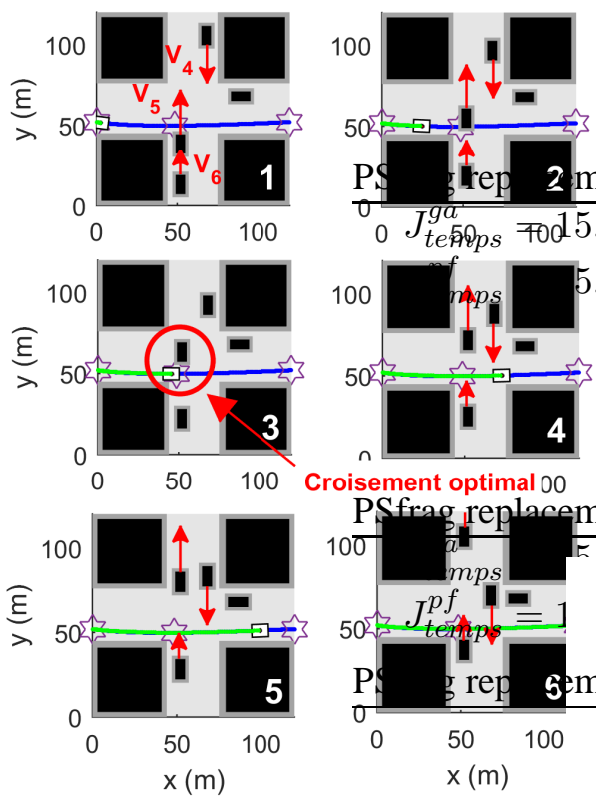

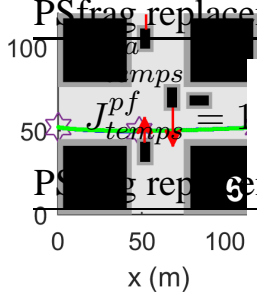

(a)
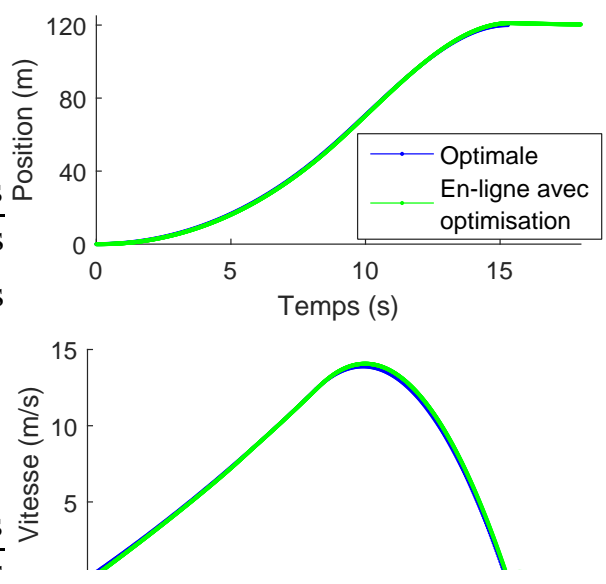

$J_{\text {temps }}^{g a}=15.29 \mathrm{~s}$

$J_{\text {temps }}^{p f}=15.34 \mathrm{~s}$

(b)

Figure 7. (a) Trajectoire en temps réel comparée à la trajectoire optimale, dans le cas d'un scénario de croisement (b) courbes de position et de vitesse correspondantes

\section{Conclusion}

Les algorithmes génétiques, une méthode aujourd'hui répandue en optimisation, sont utilisées dans cette étude pour améliorer la méthode des champs de potentiel dans une optique de planification de trajectoire. En ajoutant de l'anticipation, la méthode est rendue moins simplement réactive et plus prédictive, de sorte que l'évitement des obstacles peut se faire de façon optimale. Cela est rendu possible car le véhicule connaît toujours, au moins partiellement, son environnement proche, par le biais des capteurs à bord et les cartes fournies.

Dans cette perspective, des critères d'optimisation sont définis, et agrégés avec des coefficients de pondération. De plus des scénarios de test sont créés comportant des obstacles mobiles. Ensuite, une cible dynamique optimale est ajoutée créant une trajectoire optimale à suivre par l'EGV, permettant non seulement l'optimisation du chemin spatial mais également du mouvement le long de ce chemin. Le volume du véhicule est considéré également, de façon à l'inclure dans l'optimisation. L'effet de l'optimisation est mesuré qualitativement grâce aux simulations, et la prise en compte du mouvement des obstacles est mesurée quantitativement par le critère de temps.

Dans des travaux futurs, la méthode doit être améliorée pour que l'EGV subisse moins de pics d'accélération, qui ne permettent pas une comparaison quantitative des critères optimaux avec ceux mesurés en-ligne. De plus, en situation réelle, le véhicule se déplace et découvre un nouvel environnement en permanence, rendant la trajectoire localement sous-optimale. Cela amène la nécessité d'un recalcul périodique de la trajectoire, avec un temps de calcul très inférieur à la période de calcul. Cette étude pourrait également être étendue par l'ajout de la dynamique réelle du véhicule dans l'optimisation ainsi qu'un modèle dynamique du conducteur donnant différents jeux de pondérations pour le critère global. Les ordres des obstacles pourraient également être calculés en fonction de leur accélération. 


\section{Annexe : Valeurs des paramètres}

Les valeurs des paramètres utilisés dans l'étude sont données dans le Tableau 7.4.

\begin{tabular}{|c|c|c|}
\hline Nom du paramètre & Fonction & Valeur \\
\hline 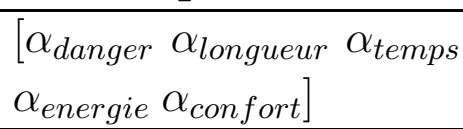 & Pondération des critères & {$\left[\begin{array}{llllll}0.35 & 0.2 & 0.2 & 0.15 & 0.1\end{array}\right]$} \\
\hline$M$ & Masse du véhicule & $900 \mathrm{~kg}$ \\
\hline$n_{\text {interp }}$ & $\begin{array}{l}\text { Nombre de points constituant la fonction } \\
\text { spline }\end{array}$ & 200 \\
\hline$b_{1}$ et $b_{2}$ & Coefficients véhicule & 1000 \\
\hline$n$ & Ordre non entier des obstacles & entre 1 et 3 exclu \\
\hline$\alpha_{p}, \alpha_{v}$ & Coefficients du correcteur proportionnel & 168750,22500 \\
\hline$T_{y}$ & Taille de l'environnement suivant $y$ & $120 \mathrm{~m}$ \\
\hline$V_{1}, V_{2}$ & $\begin{array}{l}\text { Vitesse des obstacles dans le scénario de tra- } \\
\text { versée de piéton }\end{array}$ & $2,-3 \mathrm{~m} / \mathrm{s}$ \\
\hline$V_{3}$ & Vitesse d'un obstacle dans le scénario simple & $3 \mathrm{~m} / \mathrm{s}$ \\
\hline$V_{4}, V_{5}, V_{6}$ & $\begin{array}{l}\text { Vitesse des obstacles dans le scénario de croi- } \\
\text { sement }\end{array}$ & $-2.2,4.1,1.5 \mathrm{~m} / \mathrm{s}$ \\
\hline$n_{v a r}=3 K+1$ & Nombre de variables, dans cet article & $4 ; 7 ; 10 ; 13 ;$ \\
\hline popSize & $\begin{array}{l}\text { Chaque génération possède un nombre défini } \\
\text { d'individus }\end{array}$ & 200 \\
\hline crossFrac & $\begin{array}{l}\text { Proportion de la population croisée pour la } \\
\text { suivante }\end{array}$ & 0.8 \\
\hline elitCount & Nombre d'individus élites & $\operatorname{round}(0.05 \times$ popSize $)$ \\
\hline initPop & Population initiale & Diagramme de Voronoi \\
\hline nbGen & Critère d'arrêt : nombre de générations & 300 \\
\hline tolFun & $\begin{array}{l}\text { Critère d'arrêt : taux de variation de la fonc- } \\
\text { tion coût }\end{array}$ & $10^{-6}$ \\
\hline timeLim & Critère d'arrêt : temps limité & Pas de limite de temps \\
\hline
\end{tabular}

Tableau 7.4. Valeurs des paramètres utilisés

\section{Bibliographie}

Alia, C., Gilles, T., Reine, T., and Ali, C. (2015). Local trajectory planning and tracking of autonomous vehicles, using clothoid tentacles method. In IEEE Intelligent Vehicles Symposium (IV), pages 674-679, Seoul, South Korea.

Besada-Portas, E., De La Torre, L., Moreno, A., and Risco-Martín, J. (2013). On the performance comparison of multiobjective evolutionary uav path planners. Information Sciences, $238: 111-125$.

Capello, E., Guglieri, G., Quagliotti, F., and Speciale, G. (2008). Trajectory optimisation for autonomous vehicles : A miniUAV application. The 26th International Congress of the International Council of the Aeronautical Sciences.

Choi, J.-w. and Elkaim, G. H. (2008). Bézier curves for trajectory guidance. In World Congress on Engineering and Computer Science, WCECS, pages 22-24, San Francisco, USA.

Collette, Y. and Siarry, P. (2002). Optimisation multiobjectif. Editions Eyrolles, Paris, France. 
Feng, X., Sun, Q., Liu, L., and Li, M. (2014). Assessing energy consumption of high-speed trains based on mechanical energy. Procedia - Social and Behavioral Sciences, 138(0):783 - 790.

Ge, S. and Cui, Y. (2002). Dynamic motion planning for mobile robots using potential field method. Autonomous Robots, 13(3) :207-222.

Khatib, O. (1986). Real-time obstacle avoidance for manipulators and mobile robots. The international journal of robotics research, 5(1):90-98.

Latombe, J.-C. (2012). Robot motion planning, volume 124. Springer Science \& Business Media, New York, USA.

Melchior, P., Metoui, B., Poty, A., Najar, S., Oustaloup, A., and Abdelkrim, M. N. (2007). Attractive force based on fractional potential in dynamic motion planning for mobile robot. In IDETC and IEC, pages 1423-1430, Las Vegas, Nevada, USA. ASME.

Melchior, P., Orsoni, B., and Oustaloup, A. (2001). Weyl fractional potential in path planning. In IEEE European Control Conference (ECC), pages 1758-1763, Porto, Portugal. IEEE.

Mensing, F., Trigui, R., Tattegrainc, H., and Bideaux, E. (2014). Eco-conduite des véhicules : du calcul de la trajectoire idéale à son utilisation lors de l'activité de conduite. In Symposium de Génie Électrique 2014, Cachan, France.

Nilsson, N. J. (1969). A mobile automaton : An application of artificial intelligence techniques. Technical report, Sri International menlo park CA Artificial Intelligence Center.

Petit, N. and Sciarretta, A. (2011). Optimal drive of electric vehicles using an inversion-based trajectory generation approach. 18th IFAC World Congress, pages 14519-14526.

Poty, A. (2006). Planification de trajectoire dans un environnement dynamique et génération de mouvement d'ordre non entier. PhD thesis, Université Bordeaux 1.

Pruski, A. (1996). Robotique mobile : la planification de trajectoire. Hermès, Paris.

Receveur, J.-B., Victor, S., and Melchior, P. (2017). Multi-criteria trajectory optimization for autonomous vehicles. IFACPapersOnLine, 50(1) :12520-12525. 20th IFAC World Congress.

Schouwenaars, T., De Moor, B., Feron, E., and How, J. (2001). Mixed integer programming for multi-vehicle path planning. In IEEE European Control Conference (ECC), pages 2603-2608, Porto, Portugal.

Wang, T. and Cassandras, C. (2013). Optimal motion control for energy-aware electric vehicles. In IEEE International Conference on Control Applications, pages 388-393, Hyderabad, India.

Wang, Y., Ning, B., Cao, F., De Schutter, B., and Van den Boom, T. (2011). A survey on optimal trajectory planning for train operations. In IEEE International Conference on Intelligent Rail Transportation, pages 589-594, Beijing, China. 